\title{
Marine sponge compounds with antiplasmodial properties: Focus on in vitro study against Plasmodium falciparum
}

\author{
Baso Didik Hikmawan ${ }^{1}$, Subagus Wahyuono ${ }^{2}$, Erna Prawita Setyowati ${ }^{*}$ \\ ${ }^{1}$ Master of Pharmaceutical Science Program, Faculty of Pharmacy, Universitas Gadjah Mada, Yogyakarta 55281, Indonesia \\ ${ }^{2}$ Department of Pharmaceutical Biology, Faculty of Pharmacy, Universitas Gadjah Mada, Yogyakarta 55281, Indonesia
}

\begin{tabular}{l}
\hline ARTICLE INFO \\
\hline Received on: 09/10/2019 \\
Accepted on: $18 / 02 / 2020$ \\
Available online: $06 / 05 / 2020$
\end{tabular}

Key words:

Bioactive compounds, drug development, antimalarial, marine natural product, Porifera.

\begin{abstract}
Malaria continues to be a major cause of morbidity and mortality in many tropical countries. The lack of progress in drug discovery and the spread of drug resistance become the reason behind this. Porifera (sponges) is a potential source of novel bioactive compounds to provide future drugs against malaria. In this review, we summarized 243 isolated molecules belonging to 35 different genera that active against Plasmodium falciparum from published paper until March 2019. The molecules were classified into potent, good, moderate, low, and inactive based on their $\mathrm{IC}_{50}$, and among observed bioactive metabolites, there were 57 marine sponge molecules reported to act as potent antiplasmodium against various strains of $P$. falciparum including drug resistance and nondrug resistance. Table 2 represents the list of isolated compounds with "potent" antimalarial activity. The class of the listed compounds includes manzamine alkaloid, guanidine alkaloids, bispyrroloiminoquinone alkaloids, pyrroloiminoquinone alkaloids, ingamine alkaloids, bromotyrosine alkaloids, sesquiterpenoids, diterpene formamides, aminoimidazole, $\beta$-galactosylceramides, $\beta$-lactam, meroterpene, trisoxazole macrolides, peroxides, thiazine alkaloids, and sterols. With this up-to-date review, we attempt to present new perspectives for the rational discovery of novel sponge metabolites that can be used as lead compounds in antimalarial drug development.
\end{abstract}

\section{INTRODUCTION}

Malaria is the most life-threatening and infectious disease caused by Plasmodium parasites such as Plasmodium falciparum, Plasmodium ovale, Plasmodium vivax, Plasmodium malariae. Among those protozoans, $P$. falciparum is considered to be responsible for most severe diseases and most fatal cases. The World Health Organization (2018) stated in the year of 2017 that more than $99 \%$ of estimated malaria cases in the WHO African Region followed by the WHO regions of the Western Pacific (71.9\%), the Eastern Mediterranean (69\%), and Southeast Asia $(62.8 \%)$ were caused by this most prevalent malaria parasite. In the same period, the WHO reported approximately 219 million cases of malaria occurred worldwide including 435,000 deaths.

\footnotetext{
${ }^{*}$ Corresponding Author

Erna Prawita Setyowati, Department of Pharmaceutical Biology, Faculty of Pharmacy, Universitas Gadjah Mada, Yogyakarta 55281, Indonesia. E-mail:erna_prawita@ugm.ac.id
}

Nowadays, malaria continues to be a major cause of morbidity and mortality in tropical countries. It is further aggravated by an increase in a number of multidrug-resistant strains of Plasmodium accompanied by a lack of progress in the development of vaccines and drug discovery. As a consequence, the search of new agent that actives against malaria becomes urgent needs (Antony and Parija 2016; Burrows et al., 2011; Cui et al., 2015; Dondorp et al., 2000; Noedl et al., 2008).

Marine ecosystems are the largest part of the biosphere. More than $70 \%$ of the Earth's surface is covered by water, and several theories believe that the life on earth originated from the ocean. In certain marine ecosystems such as coral reefs or the deep-sea floor, scientists estimate that the diversity of marine biota is even greater than the biota inhabiting tropical rainforests. Many immotile or slow-moving marine invertebrates, which usually do not have physical protection such as shells or thorns, will produce secondary metabolites as a form of defense mechanism from the environment and other creatures in the ocean (Ebada et al., 2008). These compounds attract the attention of researchers from various fields such as chemistry, pharmacology, biology, and ecology. This 
statement is supported by the fact that the number of new bioactive constituents isolated from marine biota has been increasing in the past three decades (332 compounds were isolated in 1984, and 1490 new compounds were isolated in 2017) (Blunt et al., 2016; Carroll et al., 2019).

Exploration of secondary metabolites from marine organisms is expected to provide new active substituents against various diseases (Newman and Cragg, 2007). Several studies have managed to isolate metabolites from marine microorganisms, green, red, and brown algae, phytoplankton, Cnidaria, Bryozoa, molluscs, tunicates, echinoderms, mangroves, sponges, and itertidal plants which have proven to have pharmaceutical properties such as acetylcholinesterase inhibitor, radical scavenging activity, cytotoxicity, antimicrobial, anticancer, antitumor, hemolytic, antiinflammatory, antiparasitic, antimalarial, and antifungal (Blunt et al., 2016; D'Ambrosio et al., 1996; Fattorusso and TaglialatelaScafati 2009; Orhan et al., 2010; Rama Rao and Faulkner 2002; Setyowati et al., 2009; 2017a; 2017b).

From the perspective of drug discovery, a marine sponge is one of the invertebrate organisms which is interesting to be explored due to its potency producing new compounds (Anjum et al., 2016). The lack of physical defense of sponges resulting in secondary metabolites is estimated to vary depending on their habitats. Metabolite compounds isolated from sponges are highly diverse such as alkaloids, esters, fatty acids, glycosides, ketones, lipids, macrolides, peptides, peroxides, quinones, terpenoids, and polyketides and have shown many biological activities, in which one of them is antimalaria (Blunt et al., 2016; 2017; 2018; Carroll et al., 2019). These kinds of compounds have been found to interfere with pathogenesis at many distinct points; therefore, this can be beneficial in developing selective antimalarial drugs (Sipkema et al., 2005)

The aim of this review is to summarize compounds isolated from marine sponges which exhibit in vitro antiplasmodial properties, to identify the compounds with potent activity based on their $\mathrm{IC}_{50}$ values, and to highlight the most important functional groups of the compounds related to their potent activity against various strains of $P$. falciparum. One of the advantages of an in vitro study is that the study could thoroughly illustrate an effect of structural features of tested compounds to their activity with no interference from other factors such as biological system which can be found on in vivo study. Therefore, it can be used to generate more potent derivatives of the compounds to develop selective antimalaria drugs that work in blood-stage P. falciparum.

\section{METHOD}

A systematic search was accomplished to find all publications related to the theme until March 2019 in PubMed and Google Scholar. The keywords used to search the articles were "Plasmodium falciparum, sponge, antimalarial" or "Plasmodium falciparum, sponge, antiplasmodial." The data included in the review were primary articles in English about in vitro antimalarial study of pure compounds isolated from marine sponges against $P$. falciparum as shown in Table 1 . The articles obtained were then removed if they are review articles, conference articles, and thesis, and there are no data available to be retrieved. All the synthetic compounds derived from naturally occurring metabolites in sponge are not mentioned in this review. Variables assessed in this review include sponge species/genus, isolated compound, strain of $P$. falciparum, region/country of origin, and effect on parasite growth inhibition.

\section{EXPLORATION OF MARINE SPONGE METABOLITES FOR ANTIPLASMODIAL ASSAY}

Among marine invertebrates, a sponge is the most dominant source for discovering natural products that have been used as lead compound to develop therapeutic drugs (Perdicaris et al., 2013). However, the study done in the investigation of marine sponge metabolites for antimalarial activity is relatively low compared to those of antitumor and anticancer. From literature published until March 2019, we included 50 primary articles for the review (Table 1). We identified that 35 different genera have been studied for their antiplasmodial activities and found that the most frequently studied genera were genus Agelas, Plakortis, and Xestospongia from different locations. Although many bioactive compounds have been isolated from marine sponges (Blunt et al., 2016; 2018; Carroll et al., 2019), the evaluation of their antiplasmodial activity is still relatively low. Figure 1 shows the number of studies that have been done on the examination of in vitro antiplasmodium of isolated compounds from marine sponge.

Overall, the number of publications from year to year shows fluctuation pattern. The highest number of the published papers was in the year of 2010 with 10 articles, followed by six publications in 2009 and 2012. In regard to the number of publications from 2013 to March 2019, it seemed to be stuck at one to three studies each year. This indicates that exploration trend of marine sponge metabolites for antiplasmodial activity diminished from 31 published papers during the period of 19922010 to 21 publications during the period of 2011-March 2019. One of the reasons behind the trend is that many scientists are interested in microbiological sample investigations for marine natural product exploration including bacteria and fungi sponge associated, making the detriment of sponge-derived compounds (Carroll et al., 2019; Thomas et al., 2010).

Various ecological studies have shown that secondary metabolites produced by sponges often serve defensive purposes to protect them from threats such as predator attacks, microbial infections, biofouling, and overgrowth by other sessile organisms (Paul and Puglisi, 2004; Paul et al., 2006). Therefore, compounds isolated from the same sponge species are more likely to be different if their habitat is distinct due to the ecological response (Mani et al., 2012). Moreover, a review done by Qaralleh (2016) found out that among 27 species of genus Neopetrosia, there are only nine species which have been chemically studied thus far. These facts disclose significant opportunities to do the chemical constituent exploration from not only genus Neopetrosia but also the other genus. In terms of collection site of the sponges, Australia, Bahamas, Indonesia, and Thailand were the most explored site so far for the search of compounds which exhibit in vitro antiplasmodium ( $P$. falciparum strains). Other sponges were collected from Turkey, Vanuatu, Madagascar, Caledonia, Fiji, China, Japan, Alaska, Jamaica, Solomon Island, Puerto Rico, Papua New Guinea, and others (Table 1). 
Table 1. Summarized data of isolated compounds which have been tested for their antiplasmodial activity.

\begin{tabular}{|c|c|c|c|c|c|c|}
\hline No & Organisms & Isolated compound & Pf Strain & $\mathrm{IC}_{50}(\mu \mathrm{M})$ & Origin & Ref. \\
\hline \multirow[t]{10}{*}{1} & Acanthella klethra & Axisonitrile 3 & D6 & 0.61 & $\begin{array}{l}\text { Pelorus Island, } \\
\text { Queensland, } \\
\text { Australia }\end{array}$ & (Angerhofer et al., 1992) \\
\hline & & & W2 & 0.07 & & \\
\hline & & Axisothiocyanate 3 & D6 & 46.85 & & \\
\hline & & & W2 & 11.81 & & \\
\hline & & The eudesmane compound $\mathrm{A}^{\mathrm{a}}$ & D6 & 8.50 & & \\
\hline & & & W2 & 2.32 & & \\
\hline & & The eudesmane compound $\mathrm{B}^{\mathrm{b}}$ & D6 & 16.17 & & \\
\hline & & & W2 & 2.22 & & \\
\hline & & The eudesmane compound $\mathrm{C}^{\mathrm{c}}$ & D6 & $>37.96$ & & \\
\hline & & & W2 & $>37.96$ & & \\
\hline \multirow[t]{8}{*}{2} & Acanthostrongylophora ingens & $(+)$-8-hydroxymanzamine A & D6 & 0.03 & Papua New Guinea & (Samoylenko et al., 2009) \\
\hline & & & W2 & 0.04 & & \\
\hline & & $(+)$-manzamine A & D6 & 0.04 & & \\
\hline & & & W2 & 0.05 & & \\
\hline & & $(+)-8$-hydroxymanzamine A hydrochloride & D6 & 0.04 & & \\
\hline & & & W2 & 0.06 & & \\
\hline & & $(+)$-manzamine A hydrochloride & D6 & 0.01 & & \\
\hline & & & W2 & 0.01 & & \\
\hline \multirow[t]{21}{*}{3} & Acanthostrongylophora sp. & Manzamine A & D6 & 0.01 & $\begin{array}{l}\text { Knife Cape } \\
\text { Manado, Indonesia }\end{array}$ & (Rao et al., 2006) \\
\hline & & & W2 & 0.01 & & \\
\hline & & $(+)$-8-hydroxymanzamine A & D6 & 0.01 & & \\
\hline & & & W2 & 0.01 & & \\
\hline & & Manzamine Y & D6 & 0.74 & & \\
\hline & & & W2 & 1.50 & & \\
\hline & & Manzamine E & D6 & 6.02 & & \\
\hline & & & W2 & 8.43 & & \\
\hline & & 6-hydroxymanzamine E & D6 & 1.36 & & \\
\hline & & & W2 & 1.50 & & \\
\hline & & Manzamine F & D6 & 1.34 & & \\
\hline & & & W2 & 2.93 & & \\
\hline & & 12,34-oxamanzamine A & D6 & 8.97 & & \\
\hline & & & W2 & na & & \\
\hline & & Ent-12,34-oxamanzamine F & D6 & 1.45 & & \\
\hline & & & W2 & 1.90 & & \\
\hline & & 12,28-oxamanzamine A & D6 and W2 & na & & \\
\hline & & 12,28-oxa-8-hydroxy-manzamine A & D6 and W2 & na & & \\
\hline & & 12,34-oxamanzamine E & D6 and W2 & na & & \\
\hline & & 12,28-oxamanzamine E & D6 and W2 & na & & \\
\hline & & 12,34-oxa-6-hydroxymanzamine E & D6 and W2 & na & & \\
\hline \multirow[t]{8}{*}{4} & Acanthostrongylophora sp. & Manzamine A N-oxide & D6 & 0.02 & Manado, Indonesia & (Rao et al., 2004) \\
\hline & & & W2 & 0.02 & & \\
\hline & & 3,4-dihydromanzamine A-N-oxide & D6 & 2.82 & & \\
\hline & & & W2 & 6.53 & & \\
\hline & & Manzamine J & D6 & 2.36 & & \\
\hline & & & W2 & 1.36 & & \\
\hline & & 6-deoxymanzamine $\mathrm{X}$ & D6 & 2.30 & & \\
\hline & & & W2 & 2.48 & & \\
\hline
\end{tabular}




\begin{tabular}{|c|c|c|c|c|c|c|}
\hline No & Organisms & Isolated compound & $P f$ Strain & $\mathrm{IC}_{50}(\mu \mathrm{M})$ & Origin & Ref. \\
\hline & & Manzamine X & D6 & 1.64 & & \\
\hline & & & W2 & 3.44 & & \\
\hline & & Neo-kauluamine & D6 & 1.46 & & \\
\hline & & & W2 & 2.41 & & \\
\hline & & Ircinal A & D6 & 5.82 & & \\
\hline & & & W2 & 7.51 & & \\
\hline & & Ircinal A & D6 and W2 & na & & \\
\hline \multirow[t]{3}{*}{5} & Agelas cf. mauritiana & Agelasine J & $\mathrm{FcB} 1$ & 6.60 & Solomon Islands & (Appenzeller et al., 2008) \\
\hline & & Agelasine K & $\mathrm{FcB} 1$ & 8.30 & & \\
\hline & & Agelasine L & $\mathrm{FcB} 1$ & 18.00 & & \\
\hline \multirow[t]{3}{*}{6} & Agelas gracilis & Gracilioethers A & ItG & 28.22 & $\begin{array}{l}\text { Oshima-Shinsone, } \\
\text { Japan }\end{array}$ & (Ueoka et al., 2009) \\
\hline & & Gracilioethers B & ItG & 1.56 & & \\
\hline & & Gracilioethers C & $\mathrm{ItG}$ & 31.02 & & \\
\hline \multirow[t]{8}{*}{7} & Agelas oroides & 24-ethyl-cholest-5 $\alpha-7$-en-3- $\alpha$-ol & K1 & 38.82 & Go"kc, eada, Turkey & (Tasdemir et al., 2007) \\
\hline & & $\begin{array}{l}\text { 4,5-dibromopyrrole-2-carboxylic acid methyl } \\
\text { ester }\end{array}$ & K1 & $>176.73$ & & \\
\hline & & $\begin{array}{l}\text { 4,5-dibromopyrrole-2-carboxylic acid (free } \\
\text { base) }\end{array}$ & K1 & $>185.95$ & & \\
\hline & & 4,5-dibromopyrrole-2-carboxylic acid (salt) & K1 & 136.37 & & \\
\hline & & (E)-oroidin (free base) & K1 & 10.02 & & \\
\hline & & (E)-oroidin (salt) & $\mathrm{K} 1$ & 16.25 & & \\
\hline & & 3-amino-1-(2-aminoimidazoyl)-prop-1-ene & K1 & 53.56 & & \\
\hline & & Taurine & K1 & $>399.52$ & & \\
\hline 8 & Agelas dispar & Longamide B & K1 & 21.19 & $\begin{array}{l}\text { Little San Salvador } \\
\text { Island }\end{array}$ & (Scala et al., 2010) \\
\hline \multirow[t]{2}{*}{9} & Agelas longissima & Longamide A & K1 & $>64.53$ & $\begin{array}{l}\text { Little San Salvador } \\
\text { Island }\end{array}$ & (Scala et al., 2010) \\
\hline & & Agelongine & K1 & 32.97 & & \\
\hline \multirow[t]{4}{*}{10} & $\begin{array}{l}\text { Genus Agelas (A. conifera, A. } \\
\text { clathrodes, A. longissima, and } \\
\text { A. dispar) }\end{array}$ & Sceptrin & K1 & 17.86 & $\begin{array}{l}\text { Little San Salvador } \\
\text { Island }\end{array}$ & (Scala et al., 2010) \\
\hline & & Hymenidin & K1 & 40.43 & & \\
\hline & & Dispacamide B & K1 & 4.11 & & \\
\hline & & Dispacamide D & K1 & $>58.45$ & & \\
\hline \multirow[t]{8}{*}{11} & Aplysinella strongylata & 19-hydroxypsammaplysin E & $3 \mathrm{D} 7$ & 6.40 & $\begin{array}{l}\text { Tulamben Bay, } \\
\text { Bali, Indonesia }\end{array}$ & (Mudianta et al., 2012) \\
\hline & & Psammaplysin K & $3 \mathrm{D} 7$ & nat $10 \mu \mathrm{M}$ & & \\
\hline & & Psammaplysin L & $3 \mathrm{D} 7$ & nat $10 \mu \mathrm{M}$ & & \\
\hline & & Psammaplysin M & $3 \mathrm{D} 7$ & nat $10 \mu \mathrm{M}$ & & \\
\hline & & Psammaplysin N & $3 \mathrm{D} 7$ & nat $10 \mu \mathrm{M}$ & & \\
\hline & & 19-hydroxypsammaplysin P & $3 \mathrm{D} 7$ & nat $10 \mu \mathrm{M}$ & & \\
\hline & & Psammaplysin T & $3 \mathrm{D} 7$ & nat $10 \mu \mathrm{M}$ & & \\
\hline & & Psammaplysin V & $3 \mathrm{D} 7$ & nat $10 \mu \mathrm{M}$ & & \\
\hline 12 & Axinyssa djiferi & Axidjiferosides (mix-A, -B, -C) & $\mathrm{FcB} 1$ & 0.53 & $\begin{array}{l}\text { Senegalese coasts, } \\
\text { Keur Bamboung }\end{array}$ & (Farokhi et al., 2013) \\
\hline \multirow[t]{6}{*}{13} & Axinella verrucosa & Stevensine & K1 & 12.61 & Calvi Bay, Corsica & (Scala et al., 2010) \\
\hline & & Spongiacidin B & K1 & 3,34 & & \\
\hline & & Bromoaldisin & K1 & $>82.08$ & & \\
\hline & & Dibromopalau'amine & K1 & $1.48 \mu \mathrm{g} / \mathrm{ml}$ & & \\
\hline & & Bromopyrrolohomoarginin & K1 & $>20 \mu \mathrm{g} / \mathrm{ml}$ & & \\
\hline & & Manzacidin A & K1 & $>20 \mu \mathrm{g} / \mathrm{ml}$ & & \\
\hline
\end{tabular}




\begin{tabular}{|c|c|c|c|c|c|c|}
\hline No & Organisms & Isolated compound & Pf Strain & $\mathrm{IC}_{50}(\mu \mathrm{M})$ & Origin & Ref. \\
\hline \multirow[t]{2}{*}{14} & Biemna laboutei & Netamine K & not available & 2.40 & $\begin{array}{l}\text { Salary Bay, } \\
\text { Madagascar }\end{array}$ & (Gros et al., 2014) \\
\hline & & Mirabilin A & not available & 20.70 & & \\
\hline \multirow[t]{8}{*}{15} & Biemna laboutei & Netamine $\mathrm{O}$ & not available & 16.99 & $\begin{array}{l}\text { Salary Bay, } \\
\text { Madagascar }\end{array}$ & (Gros et al., 2015) \\
\hline & & Netamine $\mathrm{P}$ & not available & 32.62 & & \\
\hline & & Netamine Q & not available & 8.37 & & \\
\hline & & Netamine $\mathrm{H}$ & not available & na & & \\
\hline & & Netamine I & not available & na & & \\
\hline & & Netamine $\mathrm{N}$ & not available & na & & \\
\hline & & Netamine C & not available & na & & \\
\hline & & Netamine F & not available & na & & \\
\hline \multirow[t]{8}{*}{16} & Callyspongia fibrosa & $\begin{array}{l}\text { 24S-24- methyl-cholestane } 3 \beta, 6 \beta, 25 \text {-triol- } \\
25 \text {-O-acetate }\end{array}$ & $3 \mathrm{D} 7$ & 54.81 & $\begin{array}{l}\text { The Gulf of } \\
\text { Mannar, Western } \\
\text { Bay of Bengal, } \\
\text { India }\end{array}$ & (Prakasa Rao et al., 2010) \\
\hline & & & K1 & 54.02 & & \\
\hline & & $\begin{array}{l}\text { 24S-24-methyl-cholestane-3 } \beta, 5 \alpha, 6 \beta, 25 \text {-tetraol- } \\
25 \text {-monoacetate }\end{array}$ & $3 \mathrm{D} 7$ & 30.10 & & \\
\hline & & & $\mathrm{K} 1$ & 20.54 & & \\
\hline & & $\begin{array}{l}\text { 24S-24-methyl-cholestane-3 } \beta, 6 \beta, 8 \beta, 25 \text {-tetraol- } \\
\text { 25-O-acetate }\end{array}$ & $3 \mathrm{D} 7$ & 48.46 & & \\
\hline & & & K1 & 44,44 & & \\
\hline & & $\begin{array}{l}\text { 24S-24-methyl-chelestane-3 } \beta, 5 \alpha, 6 \beta, 12 \beta, 25 \text { - } \\
\text { pentaol-25-O-acetate }\end{array}$ & $3 \mathrm{D} 7$ & 48,48 & & \\
\hline & & & K1 & 47,75 & & \\
\hline \multirow[t]{2}{*}{17} & Clathria calla & Norbatzelladine L & $\mathrm{FcB} 1$ & 0.40 & $\begin{array}{l}\text { Island of } \\
\text { Martinique }\end{array}$ & (Laville et al., 2009) \\
\hline & & Clathriadic acid & & 2.30 & & \\
\hline \multirow[t]{4}{*}{18} & Cymbastela cantharella & Girolline & $\mathrm{FcB} 1$ & 0.21 & Caledonian sponge & (Benoit-Vical et al., 2008) \\
\hline & & & W2 & 0.11 & & \\
\hline & & & FcM29 & 0.13 & & \\
\hline & & & F32 & 0.08 & & \\
\hline \multirow[t]{7}{*}{19} & Cymbastela hooperi & $\begin{array}{l}(1 \mathrm{~S}, 3 \mathrm{~S}, 4 \mathrm{R}, 7 \mathrm{~S}, 8 \mathrm{~S}, 11 \mathrm{~S}, 12 \mathrm{~S}, 13 \mathrm{~S}, 15 \mathrm{R}, 20 \mathrm{R})-7- \\
\text { Formamido-20-isocyanoisocycloamphilectane }\end{array}$ & FCR3F86 & 0.58 & Not available & $\begin{array}{l}\text { (Wright and Lang-Unnasch, } \\
\text { 2009) }\end{array}$ \\
\hline & & & W2 & 1.75 & & \\
\hline & & & D6 & 2.34 & & \\
\hline & & $\begin{array}{l}(1 \mathrm{~S}, 3 \mathrm{~S}, 4 \mathrm{R}, 7 \mathrm{~S}, 8 \mathrm{~S}, 11 \mathrm{~S}, 12 \mathrm{~S}, 13 \mathrm{~S}, 15 \mathrm{R}, 20 \mathrm{R})-7,20- \\
\text { Diformamidoisocycloamphilectane }\end{array}$ & FCR3F86 & 41.05 & & \\
\hline & & $\begin{array}{l}\left(1 \mathrm{~S}^{*}, 3 \mathrm{~S}^{*}, 4 \mathrm{R}^{*}, 7 \mathrm{~S}^{*}, 8 \mathrm{~S}^{*}, 12 \mathrm{~S}^{*}, 13 \mathrm{~S}^{*}\right)-7- \\
\text { formamidocycloamphilect-11(20)-ene }\end{array}$ & FCR3F86 & na & & \\
\hline & & $\begin{array}{l}\left(1 \mathrm{R}^{*}, 3 \mathrm{~S}^{*}, 4 \mathrm{R}^{*}, 7 \mathrm{~S}^{*}, 8 \mathrm{~S}^{*}, 12 \mathrm{~S}^{*}, 13 \mathrm{~S}^{*}\right)-7- \\
\text { formamidoamphilecta-11(20),14-diene }\end{array}$ & FCR3F86 & na & & \\
\hline & & $\begin{array}{l}\left(1 \mathrm{~S}^{*}, 3 \mathrm{~S}^{*}, 4 \mathrm{R}^{*}, 7 \mathrm{~S}^{*}, 8 \mathrm{~S}^{*}, 12 \mathrm{~S}^{*}, 13 \mathrm{~S}^{*}\right)-7- \\
\text { formamidoamphilecta-11(20),15-diene }\end{array}$ & FCR3F86 & na & & \\
\hline 20 & Desmapsamma anchorata & $\begin{array}{l}\text { sulfated } \\
\text { polysaccharides }\end{array}$ & $3 \mathrm{D} 7$ & $66.3 \mu \mathrm{g} / \mathrm{ml}$ & Not available & (Marques et al., 2016) \\
\hline \multirow[t]{7}{*}{21} & $\begin{array}{l}\text { Diacarnus } \\
\text { megaspinorhabdosa }\end{array}$ & Diacarnuperoxide M & W2 & 4.20 & Xisha Islands & (Yang et al., 2010) \\
\hline & & & D6 & 5.60 & & \\
\hline & & Diacarnuperoxide $\mathrm{N}$ & W2 & 3.00 & & \\
\hline & & & D6 & 6.60 & & \\
\hline & & $(+)-2,3,6$-epihurghaperoxide & W2 & 1.60 & & \\
\hline & & & D6 & 2.20 & & \\
\hline & & $(+)-2,3,6$-epihurghaperoxide acid & W2 & 4.90 & & \\
\hline
\end{tabular}




\begin{tabular}{|c|c|c|c|c|c|c|}
\hline No & Organisms & Isolated compound & Pf Strain & $\mathrm{IC}_{50}(\mu \mathrm{M})$ & Origin & Ref. \\
\hline & & & D6 & 7.30 & & \\
\hline & & (-)-muqubilin $\mathrm{A}$ & W2 & 5.60 & & \\
\hline & & & D6 & 8.60 & & \\
\hline & & Nuapapuin A & W2 & 5.50 & & \\
\hline & & & D6 & 8.10 & & \\
\hline & & Diacarperoxide A & W2 & 1.90 & & \\
\hline & & & D6 & 2.00 & & \\
\hline \multirow[t]{6}{*}{22} & Fascaplysinopsis reticulata & 8-oxo-tryptamine & $3 \mathrm{D} 7$ & 50.52 & $\begin{array}{l}\text { Passe Bateau, } \\
\text { Mayotte }\end{array}$ & (Campos et al., 2019) \\
\hline & & $\begin{array}{l}\text { (E) and (Z)-6-bromo-20-demethyl-30-N- } \\
\text { methylaplysinopsin }\end{array}$ & $3 \mathrm{D} 7$ & 24.01 & & \\
\hline & & 6,6'-bis-(debromo)-gelliusine $\mathrm{F}$ & $3 \mathrm{D} 7$ & na & & \\
\hline & & 6-bromo-8,1'-dihydro-isoplysin A & $3 \mathrm{D} 7$ & na & & \\
\hline & & 5,6-dibromo-8,1'-dihydro-isoplysin A & $3 \mathrm{D} 7$ & na & & \\
\hline & & tryptamine & $3 \mathrm{D} 7$ & na & & \\
\hline \multirow[t]{2}{*}{23} & Hyattella sp. & psammaplysin $\mathrm{G}$ & $\operatorname{Dd} 2$ & $\begin{array}{l}98 \% \text { iotga } \\
40 \mu \mathrm{M}\end{array}$ & $\begin{array}{l}\text { Hervey Bay, } \\
\text { Sponge Garden, } \\
\text { Queensland, } \\
\text { Australia }\end{array}$ & (Yang et al., 2010) \\
\hline & & psammaplysin F & $\operatorname{Dd} 2$ & 1.40 & & \\
\hline 24 & Hymeniacidon sp & monamphilectine A & W2 & 0.60 & $\begin{array}{l}\text { Mona Island, Puerto } \\
\text { Rico }\end{array}$ & (Avilés and Rodríguez, 2010) \\
\hline \multirow[t]{4}{*}{25} & Hyrtios cf. erecta & homofascaplysin A & K1 & 0.04 & Fiji & (Kirsch et al., 2000) \\
\hline & & & NF54 & 0.07 & & \\
\hline & & fascaplysin & K1 & 0.16 & & \\
\hline & & & NF54 & 0.11 & & \\
\hline \multirow[t]{3}{*}{26} & Hyrtios erectus & smenotronic acid & $\operatorname{Dd} 2$ & 3.51 & $\begin{array}{l}\text { Chuuk Island, } \\
\text { Federated States of } \\
\text { Micronesia }\end{array}$ & (Ju et al., 2018) \\
\hline & & ilimaquinone & $\mathrm{Dd} 2$ & 2.11 & & \\
\hline & & pelorol & $\operatorname{Dd} 2$ & 0.80 & & \\
\hline \multirow[t]{5}{*}{27} & Ircinia sp. & tryptophol & K1 & 31.51 & Aegean Sea, Turkey & (Orhan et al., 2010) \\
\hline & & $\begin{array}{l}\text { 4-hydroxy-3-tetraprenyl-phenylacetic } \\
\text { acid }\end{array}$ & K1 & 7.77 & & \\
\hline & & demethylfurospongin-4 & $\mathrm{K} 1$ & 32.23 & & \\
\hline & & dorisenone D & K1 & 1.03 & & \\
\hline & & $11 \beta$-acetoxyspongi-12-en-16-one & K1 & 3.02 & & \\
\hline \multirow[t]{6}{*}{28} & Genus Latrunculia & discorhabdins A & D6 & 0.05 & Aleutian Islands & (Na et al., 2010) \\
\hline & (later identified as Latrunculia & & W2 & 0.05 & & \\
\hline & $\begin{array}{l}\text { (L.) hamanni sp. nov. (Kelly } \\
\text { et al., 2016)) }\end{array}$ & discorhabdins $\mathrm{C}$ & D6 & 2.80 & & \\
\hline & & & W2 & 2 & & \\
\hline & & dihydrodiscorhabdin $\mathrm{C}$ & D6 & 0.17 & & \\
\hline & & & W2 & 0.13 & & \\
\hline \multirow[t]{2}{*}{29} & Lendenfeldia dendyi & Four polybromidated diphenyl ethers ${ }^{\mathrm{d}}$ & D6 & na & Papua New Guinea & (Radwan et al., 2015) \\
\hline & & & W2 & na & & \\
\hline \multirow[t]{2}{*}{30} & Mycophora sp. & Crambescidin 800 & FCR3 & 0.24 & Not available & (Lazaro et al., 2006) \\
\hline & & & 3D7 & 0.16 & & \\
\hline \multirow[t]{4}{*}{31} & Monanchora arbuscula & norbatzelladine A & $\mathrm{FcB} 1$ & 0.20 & $\begin{array}{l}\text { island of } \\
\text { Martinique }\end{array}$ & (Laville et al., 2009) \\
\hline & & dinorbatzelladine A & $\mathrm{FcB} 1$ & 0.90 & & \\
\hline & & dinordehydrobatzelladine B & $\mathrm{FcB} 1$ & 0.80 & & \\
\hline & & dihomodehydrobatzelladine $\mathrm{C}$ & FcB1 & 4.50 & & \\
\hline
\end{tabular}




\begin{tabular}{|c|c|c|c|c|c|c|}
\hline No & Organisms & Isolated compound & Pf Strain & $\mathrm{IC}_{50}(\mu \mathrm{M})$ & Origin & Ref. \\
\hline & & batzelladine A & $\mathrm{FcB} 1$ & 0.30 & & \\
\hline & & batzelladine L & $\mathrm{FcB} 1$ & 0.30 & & \\
\hline & & ptilomycalin A & $\mathrm{FcB} 1$ & 0.10 & & \\
\hline \multirow[t]{6}{*}{32} & Monanchora unguiculata & Unguiculin A & $3 \mathrm{D} 7$ & 12.89 & $\begin{array}{l}\text { Mitsio Islands, } \\
\text { Madagascar }\end{array}$ & (Campos et al., 2019) \\
\hline & & Ptilomycalin E & $3 \mathrm{D} 7$ & 0.35 & & \\
\hline & & Ptilomycalin F & $3 \mathrm{D} 7$ & 0.23 & & \\
\hline & & Ptilomycalins G $+\mathrm{H}$ & $3 \mathrm{D} 7$ & 0.46 & & \\
\hline & & Crambescidin 800 & $3 \mathrm{D} 7$ & 0.52 & & \\
\hline & & Fromiamycalin & $3 \mathrm{D} 7$ & 0.24 & & \\
\hline \multirow[t]{12}{*}{33} & New Caledonian Sponge & Alisiaquinones A & FcMC29 & 8.50 & $\begin{array}{l}\text { the Norfolk Rise } \\
\text { (New Caledonia) }\end{array}$ & (Desoubzdanne et al., 2008) \\
\hline & & & $\mathrm{FcB} 1$ & 7.40 & & \\
\hline & & & F32 & 9.10 & & \\
\hline & & Alisiaquinones B & FcMC29 & 2.60 & & \\
\hline & & & $\mathrm{FcB} 1$ & 8.40 & & \\
\hline & & & F32 & 7.10 & & \\
\hline & & Alisiaquinones $\mathrm{C}$ & FcMC29 & 0.08 & & \\
\hline & & & $\mathrm{FcB} 1$ & 0.21 & & \\
\hline & & & F32 & 0.15 & & \\
\hline & & Alisioaquinol & FcMC29 & 7.90 & & \\
\hline & & & $\mathrm{FcB} 1$ & 6.40 & & \\
\hline & & & F32 & 9.90 & & \\
\hline \multirow[t]{6}{*}{34} & Pachastrissa nux & Kabiramide J & $\mathrm{K} 1$ & 0.31 & $\begin{array}{l}\text { Koh-Tao, Surat- } \\
\text { Thani Province and } \\
\text { Chumphon Islands } \\
\text { National Park, } \\
\text { Chumphon } \\
\text { Province, Thaland }\end{array}$ & (Sirirak et al., 2011) \\
\hline & & Kabiramide K & $\mathrm{K} 1$ & 0.39 & & \\
\hline & & Kabiramide B & $\mathrm{K} 1$ & 1.67 & & \\
\hline & & Kabiramide C & $\mathrm{K} 1$ & 4.79 & & \\
\hline & & Kabiramide D & K1 & 1.87 & & \\
\hline & & Kabiramide G & $\mathrm{K} 1$ & na & & \\
\hline \multirow[t]{2}{*}{35} & Pachastrissa nux & Kabiramide L & K1 & 2.60 & $\begin{array}{l}\text { Chumphon Islands } \\
\text { National Park, } \\
\text { Thailand }\end{array}$ & (Sirirak et al., 2011) \\
\hline & & Kabiramide I & K1 & 4.50 & $\begin{array}{l}\text { Koh Tao, Surat } \\
\text { Thani Province, } \\
\text { Thailand }\end{array}$ & \\
\hline \multirow[t]{6}{*}{36} & Petrosid Ng5 Sp5 & Ingamine A & D6 & 0.20 & Not available & (Fattorusso et al., 2010) \\
\hline & & & W2 & 0.16 & & \\
\hline & & 22(S)-hydroxyingamine A & D6 & 0.47 & & \\
\hline & & & W2 & 0.30 & & \\
\hline & & Dihydroingenamine D & D6 & 0.18 & & \\
\hline & & & W2 & 0.30 & & \\
\hline \multirow[t]{6}{*}{37} & Plakortis cfr. simplex & Manadoperoxide A & D10 & 6.88 & $\begin{array}{l}\text { Bunaken Marine } \\
\text { Park of Manado, } \\
\text { Indonesia }\end{array}$ & (Fattorusso et al., 2010) \\
\hline & & & W2 & 3.74 & & \\
\hline & & Manadoperoxide B & D10 & 6.76 & & \\
\hline & & & W2 & 3.69 & & \\
\hline & & Manadoperoxide C & D10 & 4.54 & & \\
\hline & & & W2 & 2.33 & & \\
\hline
\end{tabular}




\begin{tabular}{|c|c|c|c|c|c|c|}
\hline No & Organisms & Isolated compound & Pf Strain & $\mathrm{IC}_{50}(\mu \mathrm{M})$ & Origin & Ref. \\
\hline & & Manadoperoxide D & D10 & 10.38 & & \\
\hline & & & W2 & 7.93 & & \\
\hline \multirow[t]{5}{*}{38} & Plakortis halichondrioides & Epiplakinic acid F methyl ester & W2 & 0.01 & $\begin{array}{l}\text { Mona Island, Puerto } \\
\text { Rico }\end{array}$ & $\begin{array}{l}\text { (Jiménez-Romero et al., } \\
\text { 2010) }\end{array}$ \\
\hline & & Epiplakinidioic acid & W2 & 0.95 & & \\
\hline & & Epiplakinic acid $\mathrm{F}$ & W2 & 7.93 & & \\
\hline & & Plakortolide J & W2 & na & & \\
\hline & & Plakortolide F & W2 & na & & \\
\hline \multirow[t]{8}{*}{39} & Plakortis lita & Thiaplakortones A & $3 \mathrm{D} 7$ & 0.05 & $\begin{array}{l}\text { Melville Passage, } \\
\text { Tydeman Reef, } \\
\text { Queensland, } \\
\text { Australia }\end{array}$ & (Davis et al., 2012) \\
\hline & & & $\operatorname{Dd} 2$ & 0.01 & & \\
\hline & & Thiaplakortones B & $3 \mathrm{D} 7$ & 0.65 & & \\
\hline & & & $\operatorname{Dd} 2$ & 0.09 & & \\
\hline & & Thiaplakortones C & 3D7 & 0.31 & & \\
\hline & & & $\operatorname{Dd} 2$ & 0.17 & & \\
\hline & & Thiaplakortones D & 3D7 & 0.28 & & \\
\hline & & & $\mathrm{Dd} 2$ & 0.16 & & \\
\hline \multirow[t]{6}{*}{40} & Plakortis simplex & Plakortin & D10 & 1.26 & $\begin{array}{l}\text { Berry Island } \\
\text { (Bahamas) }\end{array}$ & (Fattorusso, 2002) \\
\hline & & & W2 & 0.73 & & \\
\hline & & Dihydroplakortin & D10 & 1.12 & & \\
\hline & & & W2 & 0.76 & & \\
\hline & & Plakortide E & D10 & na & & \\
\hline & & & W2 & na & & \\
\hline \multirow[t]{4}{*}{41} & Plakortis sp. & Plakortide F & D6 & 1.35 & $\begin{array}{l}\text { Discovery Bay, } \\
\text { Jamaica }\end{array}$ & $\begin{array}{l}\text { (Gochfeld and Hamann, } \\
\text { 2001) }\end{array}$ \\
\hline & & & W2 & 1.10 & & \\
\hline & & Plakortone G & D6 & 15.09 & & \\
\hline & & & W2 & 17.10 & & \\
\hline \multirow[t]{3}{*}{42} & Genus Pseudoceratina & Psammaplysin H & 3D7 & 0.41 & Not available & (Xu et al., 2011) \\
\hline & & Psammaplysin G & $3 \mathrm{D} 7$ & 5.22 & & \\
\hline & & Psammaplysin F & 3D7 & 1.92 & & \\
\hline \multirow[t]{5}{*}{43} & Pseudoceratina sp. & Ceratinadin E & $\mathrm{K} 1$ & 0.90 & Okinawa, Japan & (Kurimoto et al., 2018) \\
\hline & & & FCR3 & 0.67 & & \\
\hline & & Ceratinadin F & K1 & $>8.16$ & & \\
\hline & & Psammaplysin F & $\mathrm{K} 1$ & 5.16 & & \\
\hline & & & FCR3 & 3.35 & & \\
\hline 44 & Pseudoceratina sp. & $\begin{array}{l}\text { Methyl (2,4-dibromo-3,6-dihydroxyphenyl) } \\
\text { acetate }\end{array}$ & $\mathrm{FcB} 1$ & 12 & $\begin{array}{l}\text { Rowa islands, } \\
\text { Banks Territory } \\
\text { (Vanuatu) }\end{array}$ & (Lebouvier et al., 2009) \\
\hline \multirow[t]{10}{*}{45} & Smenospongia aurea & 6'-chloroaureol & D6 & 9.74 & $\begin{array}{l}\text { Discovery Bay, } \\
\text { Jamaica }\end{array}$ & (Hu et al., 2002) \\
\hline & & Isoplysin A & D6 & 3.54 & & \\
\hline & & 6-bromo-2'-de-N-methylaplysinopsin & D6 & 3.45 & & \\
\hline & & 6-bromoaplysinopsin & D6 & 1.02 & & \\
\hline & & Makaluvamine O & D6 & 3.52 & & \\
\hline & & Aureol & D6 & na & & \\
\hline & & Aureol acetate & D6 & na & & \\
\hline & & 2'-de-N-methylaplysinopsin & D6 & na & & \\
\hline & & N-3'-methylaplysinopsin & D6 & na & & \\
\hline & & N-3'-ethylaplysinopsin & D6 & na & & \\
\hline
\end{tabular}




\begin{tabular}{|c|c|c|c|c|c|c|}
\hline No & Organisms & Isolated compound & Pf Strain & $\mathrm{IC}_{50}(\mu \mathrm{M})$ & Origin & Ref. \\
\hline \multirow[t]{8}{*}{46} & Spongia sp. & Squalene & K1 & $2.82 \mu \mathrm{M}$ & Aegean Sea, Turkey & (Orhan et al., 2010) \\
\hline & & Furonospinulosin-1 & $\mathrm{K} 1$ & $31.53 \mu \mathrm{M}$ & & \\
\hline & & Furospongine 1 & K1 & $42.42 \mu \mathrm{M}$ & & \\
\hline & & 2-(hexaprenylmethyl)-2-methylchromenol & K1 & $>34.19 \mu \mathrm{M}$ & & \\
\hline & & Heptaprenyl-p-quinol & K1 & $>33.28 \mu \mathrm{M}$ & & \\
\hline & & 12-epi-deoxoscalarin & K1 & $17.37 \mu \mathrm{M}$ & & \\
\hline & & 4-hydroxy-3-octaprenylbenzoic acid & K1 & $2.29 \mu \mathrm{M}$ & & \\
\hline & & furospinulosin-2 & K1 & $8.30 \mu \mathrm{M}$ & & \\
\hline 47 & Spongosorites sp. & Nortopsentin A & $3 \mathrm{D} 7$ & 0.46 & Lucaya, Bahamas & (Alvarado et al., 2013) \\
\hline \multirow[t]{6}{*}{48} & Stylissa caribica & Stevensin & D6 & 4.65 & $\begin{array}{l}\text { Columbus Park, } \\
\text { Jamaica }\end{array}$ & (Mohammed et al., 2006) \\
\hline & & oroidin & D6 & 3.08 & & \\
\hline & & Stylisin 1 & D6 & na & & \\
\hline & & Stylisin 2 & D6 & na & & \\
\hline & & Phakellistatin 13 & D6 & na & & \\
\hline & & sceptrin & D6 & na & & \\
\hline \multirow[t]{4}{*}{49} & Stylissa cf. massa & $\begin{array}{l}\text { 8-isocyanato-15-formamidoamphilect-11(20)- } \\
\text { ene }\end{array}$ & K1 & 8.85 & $\begin{array}{l}\text { Koh-Tao, Surat- } \\
\text { Thani Province, } \\
\text { Thailand }\left(10^{\circ} 7.569^{\prime}\right. \\
\left.\text { N, } 99^{\circ} 48.665^{\prime} \mathrm{E}\right)\end{array}$ & $\begin{array}{l}\text { (Chanthathamrongsiri et al., } \\
\text { 2012) }\end{array}$ \\
\hline & & $\begin{array}{l}\text { 8-isothiocyanato-15-formamidoamphilect- } \\
\text { 11(20)-ene }\end{array}$ & K1 & 8.07 & & \\
\hline & & 8-isocyano-15-formamidoamphilect-11(20)-ene & K1 & 0.52 & & \\
\hline & & 7-formamidoamphilect-11(20),15-diene & K1 & na & & \\
\hline \multirow[t]{24}{*}{50} & Suberea ianthelliformis & Araplysillin I & $\mathrm{FcB} 1$ & 4.5 & $\begin{array}{l}\text { Anuta Paina Island } \\
\text { (Malaita) }\end{array}$ & (Mani et al., 2012) \\
\hline & & & $3 \mathrm{D} 7$ & 4.6 & & \\
\hline & & Araplysillin II & $\mathrm{FcB} 1$ & 34.2 & & \\
\hline & & Araplysillin N20-formamide & $\mathrm{FcB} 1$ & 3.6 & & \\
\hline & & & 3D7 & 7.0 & & \\
\hline & & Araplysillin IV & $\mathrm{FcB} 1$ & 27.6 & & \\
\hline & & Araplysillin V & $\mathrm{FcB} 1$ & 50.5 & & \\
\hline & & Araplysillin VI & $\mathrm{FcB} 1$ & 37.4 & & \\
\hline & Suberea ianthelliformis & Aerophobin I & $\mathrm{FcB} 1$ & 59.0 & New Georgia Island & (Mani et al., 2012) \\
\hline & & Aerophobin II & $\mathrm{FcB} 1$ & 24.9 & & \\
\hline & & & $3 \mathrm{D} 7$ & 19.9 & & \\
\hline & & Purealidin Q & $\mathrm{FcB} 1$ & 3.6 & & \\
\hline & & Araplysillin N20-hydroxyformamide & $\mathrm{FcB} 1$ & 5.0 & & \\
\hline & & & $3 \mathrm{D} 7$ & 4.1 & & \\
\hline & Suberea ianthelliformis & Aerothionin & $\mathrm{FcB} 1$ & 3.4 & $\begin{array}{l}\text { North West of } \\
\text { Nggela Island }\end{array}$ & (Mani et al., 2012) \\
\hline & & & $3 \mathrm{D} 7$ & 4.2 & & \\
\hline & & Homoaerothionin & $\mathrm{FcB} 1$ & 2.8 & & \\
\hline & & & $3 \mathrm{D} 7$ & 4.0 & & \\
\hline & & 11,19-Dideoxyfistularin 3 & $\mathrm{FcB} 1$ & 2.1 & & \\
\hline & & & $3 \mathrm{D} 7$ & 0.9 & & \\
\hline & & 11-Hydroxyfistularin 3 & $\mathrm{FcB} 1$ & 2.1 & & \\
\hline & & & 3D7 & 2.6 & & \\
\hline & & Aplysinone D & $\mathrm{FcB} 1$ & 1.0 & & \\
\hline & & & $3 \mathrm{D} 7$ & 3.1 & & \\
\hline
\end{tabular}




\begin{tabular}{|c|c|c|c|c|c|c|}
\hline No & Organisms & Isolated compound & $P f$ Strain & $\mathrm{IC}_{50}(\mu \mathrm{M})$ & Origin & Ref. \\
\hline \multirow[t]{8}{*}{51} & Verongula rigida & Purealidin B & NF54 & $\begin{array}{c}23.2 \% \text { iotga } \\
5 \mu \mathrm{M}\end{array}$ & $\begin{array}{l}\text { Urabá Gulf, } \\
\text { Caribbean } \\
\text { Sea, Colombia } \\
\left(8^{\circ} 40^{\prime} 14^{\prime \prime} \mathrm{N},\right. \\
\left.77^{\circ} 21^{\prime} 28^{\prime \prime} \mathrm{W}\right)\end{array}$ & (Galeano et al., 2011) \\
\hline & & 11-hydroxyaerothionin & NF54 & $\begin{array}{c}8.0 \% \text { iotga } \\
5 \mu \mathrm{M}\end{array}$ & & \\
\hline & & Aeroplysinin & NF54 & $\begin{array}{c}35.3 \% \text { iotga } \\
5 \mu \mathrm{M}\end{array}$ & & \\
\hline & & Dihydroxyaerothionin & NF54 & $\begin{array}{c}7.9 \% \text { iotga } \\
5 \mu \mathrm{M}\end{array}$ & & \\
\hline & & Purealidin R & NF54 & $\begin{array}{c}7.1 \% \text { iotga } \\
5 \mu \mathrm{M}\end{array}$ & & \\
\hline & & 3,5-dibromo-N,N,N-trimethyltyraminium & NF54 & na & & \\
\hline & & 3,5-dibromo-N,N,N,O-tetramethyltyraminium & NF54 & na & & \\
\hline & & 19-deoxyfistularin 3 & NF54 & na & & \\
\hline \multirow[t]{6}{*}{52} & Xestospongia exigua & Araguspongine $\mathrm{C}$ & D6 & 1.4 & $\begin{array}{l}\text { Bayadha, Saudi } \\
\text { Arabian Red Sea } \\
\text { coast }\end{array}$ & (Orabi et al., 2002) \\
\hline & & & W2 & 0.58 & & \\
\hline & & $(+)$ - Araguspongine $\mathrm{K}$ & D6 & na & & \\
\hline & & & W2 & na & & \\
\hline & & $(+)$ - Araguspongine L & D6 & na & & \\
\hline & & & W2 & na & & \\
\hline \multirow[t]{2}{*}{53} & Xestospongia sp. & Kaimanol & $3 \mathrm{D} 7$ & 0.36 & $\begin{array}{l}\text { Kaimana, West } \\
\text { Papua, Indonesia }\end{array}$ & (Murtihapsari et al., 2019) \\
\hline & & Saringosterol & $3 \mathrm{D} 7$ & $2.50 \times 10^{-4}$ & & \\
\hline 54 & Xestospongia sp. & Xestoquinone & $\mathrm{FcB} 1$ & 3 & $\begin{array}{l}\text { Malvoror reef, } \\
\text { Vanuatu }\end{array}$ & (Laurent et al., 2006) \\
\hline \multirow[t]{16}{*}{55} & genus Xestospongia & Halenaquinone & FcB1 & $>30$ & South Pacific & (Longeon et al., 2010) \\
\hline & & & $3 \mathrm{D} 7$ & $>30$ & & \\
\hline & & 3-Ketoadociaquinone $\mathrm{A}$ & $\mathrm{FcB} 1$ & 1.08 & & \\
\hline & & & $3 \mathrm{D} 7$ & 1.67 & & \\
\hline & & 3-Ketoadociaquinone B & $\mathrm{FcB} 1$ & 3.89 & & \\
\hline & & & $3 \mathrm{D} 7$ & 4.12 & & \\
\hline & & Tetrahydrohalenaquinone A & $\mathrm{FcB} 1$ & $>29$ & & \\
\hline & & & $3 \mathrm{D} 7$ & $>29$ & & \\
\hline & & Tetrahydrohalenaquinone B & FcB1 & $>29$ & & \\
\hline & & & $3 \mathrm{D} 7$ & $>29$ & & \\
\hline & & Halenaquinol sulfate & FcB1 & $>24$ & & \\
\hline & & & $3 \mathrm{D} 7$ & $>24$ & & \\
\hline & & Xestosaprol C methylacetal & $\mathrm{FcB} 1$ & $>21$ & & \\
\hline & & & $3 \mathrm{D} 7$ & $>21$ & & \\
\hline & & Orhalquinone & $\mathrm{FcB} 1$ & 9.22 & & \\
\hline & & & $3 \mathrm{D} 7$ & 10.94 & & \\
\hline \multirow[t]{7}{*}{56} & Zyzzya sp. & Tsitsikammamine C & $3 \mathrm{D} 7$ & 0.01 & $\begin{array}{l}\text { Rodda Reef, } \\
\text { Queensland, } \\
\text { Australia }\end{array}$ & (Davis et al., 2012) \\
\hline & & & Dd2 & 0.02 & & \\
\hline & & makaluvamines J & $3 \mathrm{D} 7$ & 0.02 & & \\
\hline & & & $\operatorname{Dd} 2$ & 0.02 & & \\
\hline & & makaluvamines G & $3 \mathrm{D} 7$ & 0.04 & & \\
\hline & & & $\mathrm{Dd} 2$ & 0.04 & & \\
\hline & & makaluvamines L & $3 \mathrm{D} 7$ & 0.04 & & \\
\hline
\end{tabular}




\begin{tabular}{|c|c|c|c|c|c|c|}
\hline No & Organisms & Isolated compound & $P f$ Strain & $\mathrm{IC}_{50}(\mu \mathrm{M})$ & Origin & Ref. \\
\hline & & \multirow{3}{*}{ makaluvamines $\mathrm{K}$} & $\operatorname{Dd} 2$ & 0.02 & & \\
\hline & & & $3 \mathrm{D} 7$ & 0.40 & & \\
\hline & & & $\operatorname{Dd} 2$ & 0.30 & & \\
\hline & & \multirow[t]{2}{*}{ Damirone A } & 3D7 & 1.88 & & \\
\hline & & & $\operatorname{Dd} 2$ & 0.36 & & \\
\hline & & \multirow[t]{2}{*}{ Damirone B } & $3 \mathrm{D} 7$ & 12.25 & & \\
\hline & & & $\mathrm{Dd} 2$ & 3.80 & & \\
\hline
\end{tabular}

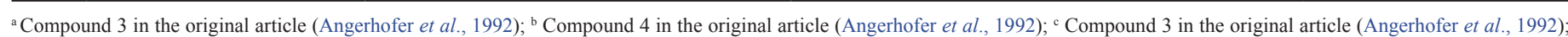

${ }^{\mathrm{d}}$ Compound 1, 2, 3, and 4 in the original article (Radwan et al., 2015) nat = not active at (inhibition of parasite growth); na = not active; iotga = inhinition of the growth at

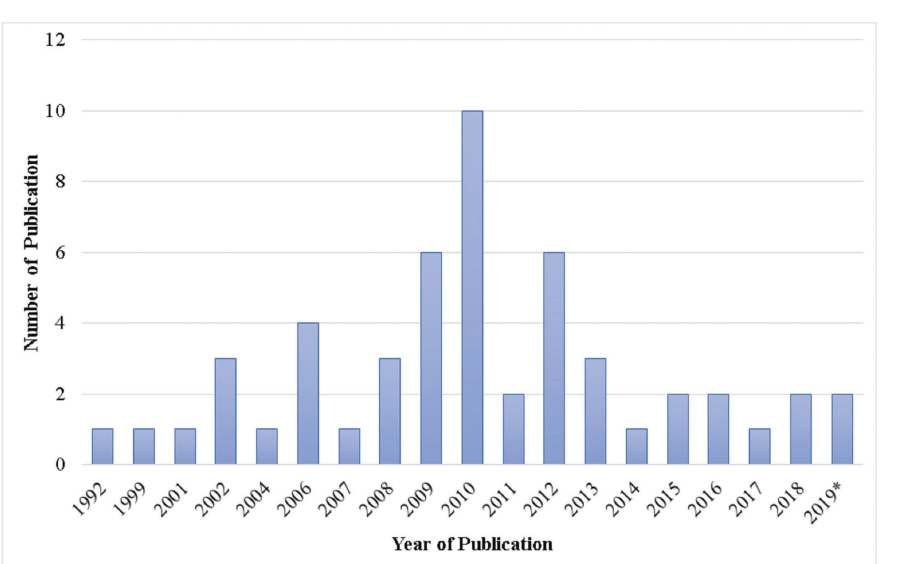

*March 2019

Figure 1. Distribution of conducted studies about marine sponge metabolite exploration for in vitro antiplasmodium.

\section{CLASSIFICATION OF ANTIPLASMODIAL ACTIVITY OF ISOLATED COMPOUND FROM SPONGES}

In this review, we give an overview of the bioactive metabolites recently isolated from marine sponges that have shown activity in in vitro study against $P$. falciparum. To compare the $\mathrm{IC}_{50}$ values, the units in $\mu \mathrm{g} / \mathrm{ml}$ and $\mathrm{nM}$ were converted to $\mu \mathrm{M}$. All the isolated compounds were then classified based on their $\mathrm{IC}_{50}$ values by following the definition of Batista et al. (2009), who grouped compounds into potent activity: $\mathrm{IC}_{50}<1 \mu \mathrm{M}$, good activity: $\mathrm{IC}_{50}$ of $1-20 \mu \mathrm{M}$, moderate activity: $\mathrm{IC}_{50}$ of $20-100 \mu \mathrm{M}$, low activity: $\mathrm{IC}_{50}$ of $100-200 \mu \mathrm{M}$, and inactive: $\mathrm{IC}_{50}>200 \mu \mathrm{M}$ (Batista et al., 2009). To be noted, the mechanism of the in vitro continuous cultures of $P$. falciparum approach is only related to the inhibition of growth in erythrocytic stages of the parasite (Chin et al., 1979). Consequently, this $\mathrm{IC}_{50}$-based classification would exclude compounds that may have other specific mechanism of action. It would be wise to re-evaluate "not active compounds" with other assay or holistic approach such as the reverse pharmacology technique (Simoes-Pires et al., 2014).

As shown in Figure 2, among observed bioactive metabolites, there were 57 different compounds that have potent activity, 101 with good activity, and 26 compounds with moderate activity against various strains of $P$. falciparum. Some of the compounds could not be classified because, in the highest tested concentration, their activity was low or inactive and some reports use inhibition concentration instead of $\mathrm{IC}_{50}$, making it incomparable. In regard to the dependency of $\mathrm{IC}_{50}$ to plasmodium

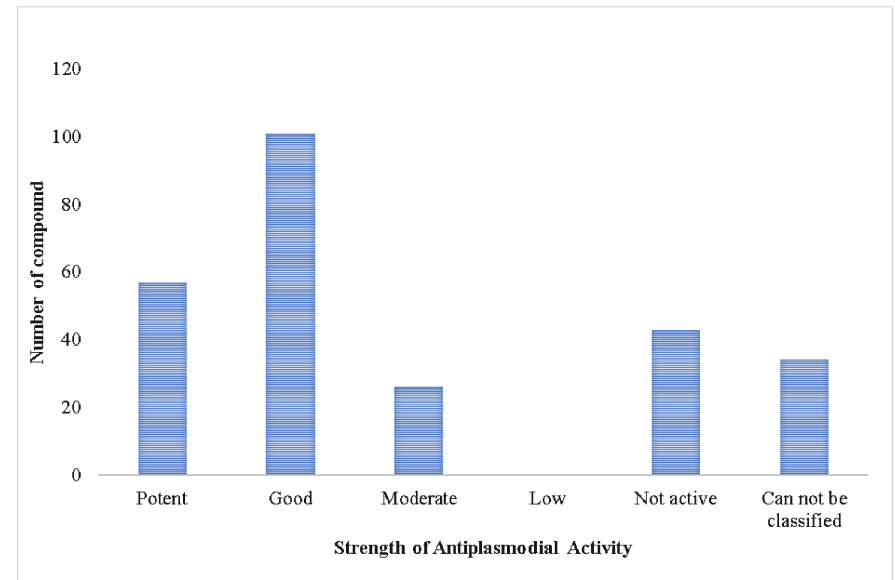

Figure 2. Classification of the isolated compounds activity according to their $\mathrm{IC}_{50}$ values.

strains, it seems that antiplasmodial activity of some isolated compounds did not depend on chloroquine/drug sensitivity of the strain (Fattorusso et al., 2010; Longeon et al., 2010; Mani et al., 2012).

The class of compounds which exhibit potent antiplasmodial activity includes manzamine alkaloid (Rao et al., 2004; 2006; Samoylenko et al., 2009), guanidine alkaloids (Campos et al., 2017; Laville et al.,2009), bispyrroloiminoquinone alkaloid (Davis et al., 2012), pyrroloiminoquinone alkaloids ( $\mathrm{Na}$ et al., 2010), ingamine alkaloids (Ilias et al., 2012), sesquiterpenoids (Angerhofer et al., 1992), diterpene formamides (Wright and Lang-Unnasch, 2009), aminoimidazole (Benoit-Vical et al., 2008), $\beta$-galactosyl ceramides (Farokhi et al., 2013), $\beta$-lactam (Avilés and Rodríguez, 2010), meroterpene (Desoubzdanne et al., 2008), trisoxazole macrolides (Sirirak et al., 2011), peroxides, thiazine alkaloids (Davis et al., 2012), bromotyrosine alkaloids (Kurimoto et al., 2018; Xu et al., 2011), and sterols (Murtihapsari et al., 2019).

\section{FUNCTIONAL GROUP IN POTENT ANTIPLASMODIAL ACTIVITY}

Some marine isonitriles show various biological activities such as antimalarial, antitubercular, antifouling, and antiplasmodial effect. Marine isonitriles differ from terrestrial isonitriles in terms of their biosynthetic pathways. Most of the marine compounds containing isonitrile were derived from terpenoid, whereas terrestrial isonitriles originate from $\alpha$-amino acids (Emsermann 
Table 2. List of isolated compounds with potent antiplasmodial activity based on $\mathrm{IC}_{50}$ measurement.

\begin{tabular}{|c|c|c|}
\hline No. & Isolated Compound & P. falciparum strain \\
\hline 1 & Axisonitrile 3 & D6 and W2 \\
\hline 2 & (+)-8-hydroxymanzamine A & D6 and W2 \\
\hline 3 & $(+)$-manzamine A & D6 and W2 \\
\hline 4 & (+)-8-hydroxymanzamine A hydrochloride & D6 and W2 \\
\hline 5 & $(+)$-manzamine A hydrochloride & D6 and W2 \\
\hline 6 & Manzamine A & D6 and W2 \\
\hline 7 & Manzamine Y & D6 \\
\hline 8 & Manzamine A N-oxide & D6 and W2 \\
\hline 9 & Axidjiferosides & $\mathrm{FcB} 1$ \\
\hline 10 & Norbatzelladine L & $\mathrm{FcB} 1$ \\
\hline 11 & Girolline & $\begin{array}{l}\text { FcB1; W2; FcM29; } \\
\text { and F32 }\end{array}$ \\
\hline 12 & $\begin{array}{l}\text { (1S,3S,4R,7S,8S,11S,12S,13S,15R,20R)-7- } \\
\text { Formamido-20-isocyanoisocycloamphilectane }\end{array}$ & FCR3F86 \\
\hline 13 & Monamphilectine A & W2 \\
\hline 14 & Homofascaplysin A & $\mathrm{K} 1$ and NF54 \\
\hline 15 & Fascaplysin & $\mathrm{K} 1$ and NF54 \\
\hline 16 & Pelorol & $\operatorname{Dd} 2$ \\
\hline 17 & Discorhabdins A & D6 and W2 \\
\hline 18 & Dihydrodiscorhabdin C & D6 and W2 \\
\hline 19 & Crambescidin 800 & FCR3 and 3D7 \\
\hline 20 & Norbatzelladine A & $\mathrm{FcB} 1$ \\
\hline 21 & Dinorbatzelladine A & $\mathrm{FcB} 1$ \\
\hline 22 & Dinordehydrobatzelladine B & $\mathrm{FcB} 1$ \\
\hline 23 & Batzelladine A & $\mathrm{FcB} 1$ \\
\hline 24 & Batzelladine L & $\mathrm{FcB} 1$ \\
\hline 25 & Ptilomycalin A & $\mathrm{FcB} 1$ \\
\hline 26 & Ptilomycalin E & $3 \mathrm{D} 7$ \\
\hline 27 & Ptilomycalin F & 3D7 \\
\hline 28 & Ptilomycalin $\mathrm{G}+\mathrm{H}$ & $3 \mathrm{D} 7$ \\
\hline 29 & Fromiamycalin & 3D7 \\
\hline 30 & Alisiaquinone $\mathrm{C}$ & $\begin{array}{l}\text { FcMC29; FcB1; and } \\
\text { F32 }\end{array}$ \\
\hline 31 & Kabiramide $\mathbf{J}$ & K1 \\
\hline 32 & Kabiramide K & K1 \\
\hline 33 & Ingamine $\mathrm{A}$ & D6 and W2 \\
\hline 34 & 22(S)-hydroxyingamine A & D6 and W2 \\
\hline 35 & Dihydroergotamine D & D6 and W2 \\
\hline 36 & Epiplakinic acid F methyl ester & W2 \\
\hline 37 & Epiplakinidioic acid & W2 \\
\hline 38 & Thiaplakortone A & $3 \mathrm{D} 7$ and Dd2 \\
\hline 39 & Thiaplakortone B & 3D7 and Dd2 \\
\hline 40 & Thiaplakortone C & 3D7 and Dd2 \\
\hline 41 & Thiaplakortone D & 3D7 and Dd2 \\
\hline 42 & Plakortin & W2 \\
\hline 43 & Dihydroplakortin & W2 \\
\hline 44 & Psammaplysin $\mathrm{H}$ & $3 \mathrm{D} 7$ \\
\hline 45 & Ceratinadin $\mathrm{E}$ & FCR3 \\
\hline 46 & Nortopsentin A & 3D7 \\
\hline 47 & 8-isocyano-15-formamidoamphilect-11(20)-ene & $\mathrm{K} 1$ \\
\hline
\end{tabular}

\begin{tabular}{cll}
\hline No. & Isolated Compound & P. falciparum strain \\
\hline 48 & $11,19-$ Dideoxyfistularin 3 & 3D7 \\
49 & Araguspongine C & W2 \\
50 & Kaimanol & 3D7 \\
51 & Saringosterol & 3D7 \\
52 & Tsitsikammamine C & 3D7 and Dd2 \\
53 & Makaluvamine J & 3D7 and Dd2 \\
54 & Makaluvamine G & 3D7 and Dd2 \\
55 & Makaluvamine L & 3D7 and Dd2 \\
56 & Makaluvamine K & 3D7 and Dd2 \\
57 & Damirone A & Dd2 \\
\hline
\end{tabular}

et al., 2016). Axisonitrile-3 (1) is a sesquiterpene derived from chloroform fraction of sponge Acanthella klethra containing isonitrile group which appears to be crucial for activity since the corresponding isothiocyanate derivative compound 2 (moderate activity) is less active than 1 (potent activity) (Angerhofer et al., 1992). The eudesmane compounds 3 and 4 which contain isothiocyanate still showed good antiplasmodial activity, whereas the reversal of the stereochemical configuration between 4 and 5 exhibits a significant change on their antiplasmodial effect (see Figure 3).

The manzamines are a group of marine alkaloids characterized by a fused and bridged tetra- or pentacyclic ring system attached to a $\beta$-carboline moiety. Since manzamine was isolated in different genus of sponges, it is thought that manzamine is actually produced by associated microorganism. An interesting review had been done by Fattorusso and Taglialatela-Scafati (2009) who described the key role of the eight member rings as well as other functional groups that affect the antiplasmodial activity of manzamines; therefore, we will not discuss it in this review.

A mixture of new glycosphingolipids named axidjiferoside A (6), axidjiferoside $\mathrm{B}$ (7), and axidjiferoside $\mathrm{C}$ (8) shows a potent antiplasmodial activity (Figure 3 ). Compounds 6, 7 , and 8 were isolated from Senegal marine sponge Axinyssa djiferi (Farokhi et al., 2013). These compounds contain sphingolipid structure which are found in ceramide analogs, PPMP (d,1-threo1-phenyl-2-palmitoylamino-3-morpholino-1-propanol), and PDMP (1-phenyl-2-decanoylamino-3-morpholino-1-propanol). These analogs are known to inhibit the parasite sphingomyelin synthase activity and block parasite development by preventing the formation of the tubovesicular network that extends from the parasitophorous vacuole to the red cell membrane and delivers essential extracellular nutrients to the parasite (Labaied et al., 2004; Zhang et al., 2010).

Bioactive guanidine alkaloids including norbatzelladine A (9), dinorbatzelladine A (10), batzelladine A (11), dinordehydrobatzelladine B (12), norbatzelladine L (13), and batzelladine L (14) are potent against the growth of P. falciparum. The aromatization in the tricyclic core of 11 (compared to 9 and 8) did not change the antimalarial activity. Batzelladine $\mathrm{A}$, with one bicyclic and one tricyclic guanidine core, has similar properties with 9,13 , and 14 in terms of the activity against $P$. falciparum strain FcB1, where 13 and 14 have two tricyclic guanidine cores. The reduction of bicyclic core in dihomodehydrobatzelladine $\mathrm{C}$ seems to affect its activity to be less active than 9-12 (Figure 3). 


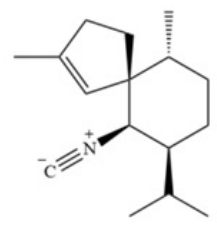

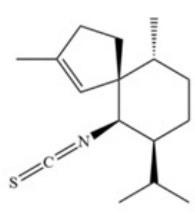

2

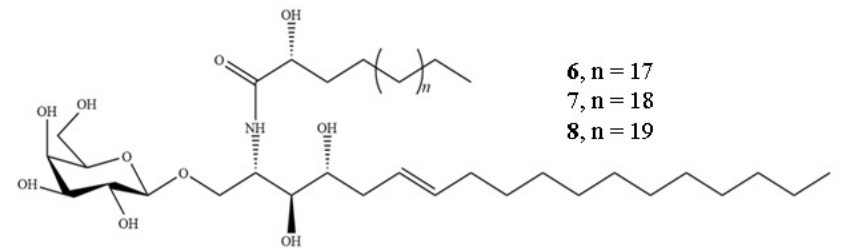<smiles>CC1CCCC2(C)CC[C@H](C(C)(C)C)C=C12</smiles>

3<smiles>C=C(C)[C@H]1CCC2(C)CCC[C@H]([AsH3])[C@H]2C1</smiles>

4

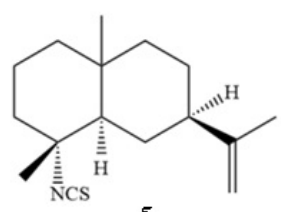

5

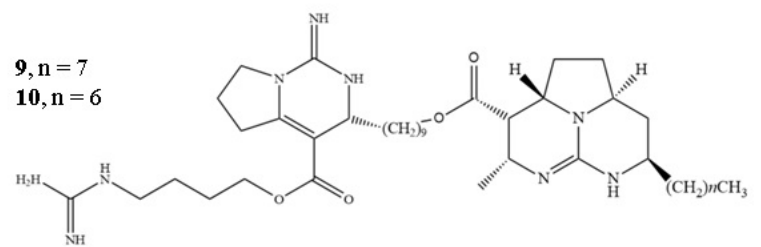

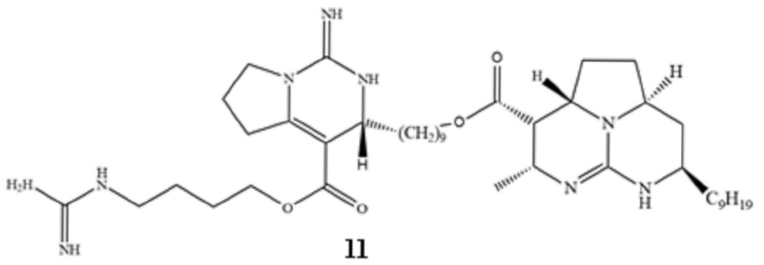

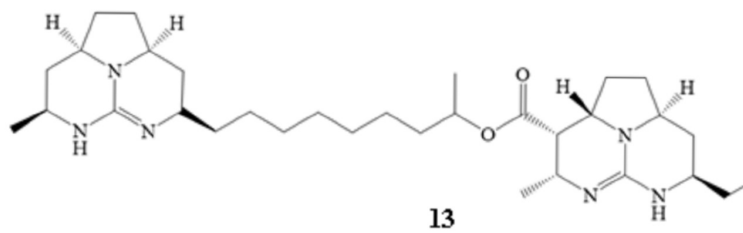

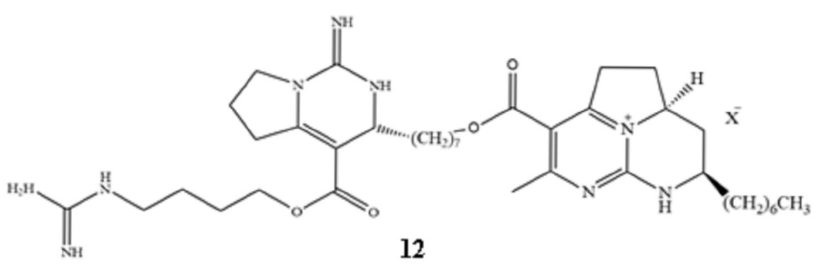<smiles>CCCCCCCCC[C@@H]1C[C@H]2CC[C@H]3[C@H](C(=O)OC(C)CCCCCCC[C@@H]4C[C@@H]5CC[C@@H]6C[C@H](C)NC(=N4)N56)[C@H](C)N=C(N1)N32</smiles>

14

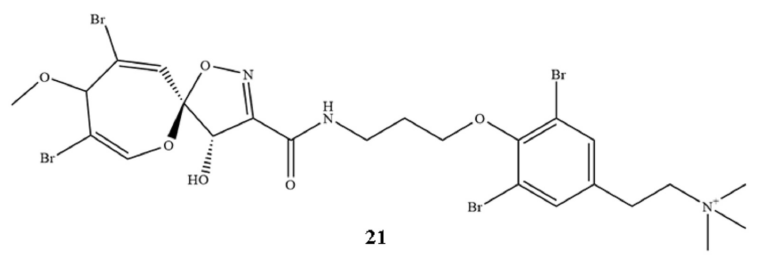

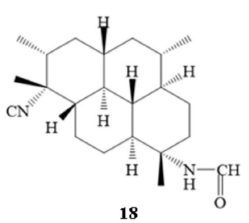

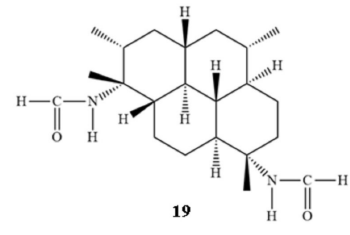<smiles>NC[C@@H](Cl)[C@H](O)c1cnc(N)s1</smiles>

17

21

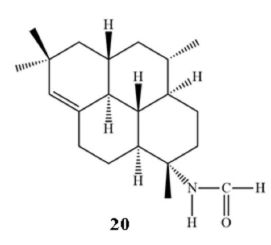<smiles>COC1=C(Br)C[C@@]2(CC(C)=NO2)OC=C1Br</smiles><smiles>CNCCc1cc(Br)c(OCCCNC(C)=O)c(Br)c1</smiles>

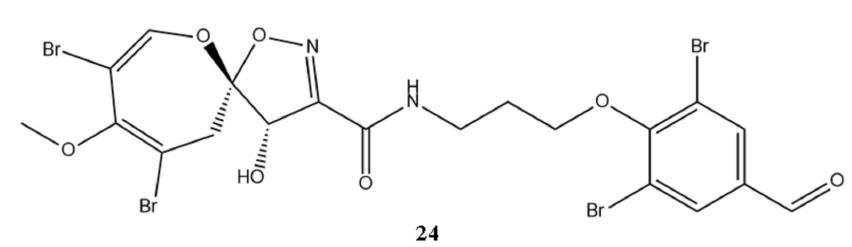

Figure 3. Structure of antimalarial compounds (Angerhofer et al., 1992; Benoit-Vical et al., 2008; Farokhi et al., 2013; Mudianta et al., 2012; Wright and Lang-Unnasch 2009; Xu et al., 2011). 
Girolline (15), 2-aminoimidazole derivative, isolated from Cymbastela cantharella showed a potent activity against $P$. falciparum strains, whereas its analogs 5-deazathiogirollines (16 and 17) were considered to be inactive (Benoit-Vical et al., 2008). This indicates that imidazole ring in 15 plays an important role in the antiplasmodial activity.

Sponge Cymbastela hooperi sp. nov. described by Soest et al. (1996) produces a plethora of chemical compounds structurally related to diterpene isonitrile derivatives which exhibit significant in vitro antimalarial activity. (1S, 3S, 4R, 7S, 8S, 11S, 12S, 13S, 15R,20R)-7-Formamido-20isocyanoisocycloamphilectane (18), $(1 S, 3 S, 4 R, 7 S, 8 S, 11 S, 12 S, 1$ $3 S, 15 R, 20 R)-7,20$-Diformamidoisocycloamphilectane (19), and $\left(1 S^{*}, 3 S^{*}, 4 R^{*}, 7 S^{*}, 8 S^{*}, 12 S^{*}, 13 S^{*}\right)-7$-Formamidocycloamphilect11(20)-ene (20) were new diterpene formamides which were isolated from C. hooperi (Figure 3). Compound 18 is a unique molecule since it contains both formamide and isonitrile functionalities where such a feature is rarely found in natural product. Based on its $\mathrm{IC}_{50}$ against $P$. falciparum $\mathrm{FCR} 3 \mathrm{~F} 86$, this substituent is classified into potent (Wright and Lang-Unnasch, 2009). The lack of isonitrile in the structure of 19 decreases the activity to be moderate. This finding is supported by the activity of compound 1 that possesses isonitrile too (Angerhofer et al., 1992).

Psammaplysin H (21) derived from sponge genus Pseudoceratina is also included in the potent activity group against $P$. falciparum $3 \mathrm{D} 7$ with $\mathrm{IC}_{50} 0.41 \mu \mathrm{M}$. This activity is more likely caused by the presence of quaternary amine in the $\mathrm{R}$ group at C-20 (see Figure 3). However, the secondary amine at the same position in psammaplysin $\mathrm{F}$ (22) reduced antimalarial activity 4-fold lower than compound 21 . In addition, when the alkyl amine is substituted with a urea at C-20 in Psammaplysin G (23), the activity decreased to have $\mathrm{IC}_{50} 5.99 \mu \mathrm{M}$ (Xu et al., 2011). Consistently, the loss of amine substituent in psammaplysin K (24) dispelled the antiplasmodial activity (Mudianta et al., 2012).

\section{CONCLUSION}

Data presented in the review indicate that marine sponges could be used as sources for lead compounds in drug discovery program including the development of non-resistance antimalarial drugs in this case. The summarized "potent" isolated compounds highlight the most promising candidates which include manzamine alkaloids, guanidine alkaloids, bispyrroloiminoquinone alkaloid, pyrroloiminoquinone alkaloids, ingamine alkaloids, sesquiterpenoids, diterpene formamides, aminoimidazole, $\beta$-galactosyl ceramides, $\beta$-lactam, meroterpene, trisoxazole macrolides, peroxides, thiazine alkaloids, bromotyrosine alkaloids, and sterols. A holistic approach for their pharmacological evaluation is still needed since in vitro $P$. falciparum assay could only evaluate a specific mechanism of action for antiplasmodium. To reproduce the compounds for their further evaluation, the possibility of bioengineering or/and bacterial fermentation could be worth.

\section{ACKNOWLEDGMENT}

The author would like to acknowledge the funding support from UGM No: 3040/UN1/DITLIT/DIT-LIT/LT/2019.

\section{REFERENCES}

Alvarado S, Roberts BF, Wright AE, Chakrabarti D. The Bis(Indolyl)Imidazole alkaloid nortopsentin A exhibits antiplasmodial activity. Antimicrob Agents Chemother, 2013; 57(5):2362-4.

Angerhofer CK, Pezzuto JM, König GM, Wright AD, Sticher O. Antimalarial activity of sesquiterpenes from the marine sponge Acanthella klethra. J Nat Prod, 1992; 55(12):1787-9.

Anjum K, Abbas SQ, Shah SAA, Akhter N, Batool S, ul Hassan SS. Marine sponges as a drug treasure. Biomol Therap, 2016; 24(4):347-62. Antony HA, Parija SC. Antimalarial drug resistance: an overview. Trop Parasitol, 2016; 6(1):30-41.

Appenzeller J, Mihci G, Martin M-T, Gallard J-F, Menou J-L, Boury-Esnault N, Hooper J, Petek S, Chevalley S, Valentin A, Zaparucha A. Agelasines J, K, and L from the Solomon Islands marine sponge Agelas $\mathrm{cf}$. mauritiana. J Nat Prod, 2008; 71(8):1451-4.

Avilés E, Rodríguez AD. Monamphilectine A, a potent antimalarial $\beta$-lactam from marine sponge Hymeniacidon sp: isolation, structure, semisynthesis, and bioactivity. Org Lett, 2010; 12(22):5290-3.

Batista R, De Jesus Silva Júnior A, De Oliveira A. Plant-derived antimalarial agents: new leads and efficient phytomedicines. Part II. Nonalkaloidal natural products. Molecules, 2009; 14(8):3037-72.

Benoit-Vical F, Saléry M, Soh P, Ahond A, Poupat C. Girolline: a potential lead structure for antiplasmodial drug research. Planta Med, 2008; 74(4):438-44.

Blunt JW, Carroll AR, Copp BR, Davis RA, Keyzers RA, Prinsep MR. Marine natural products. Nat Prod Rep, 2018; 35(1):8-53.

Blunt JW, Copp BR, Keyzers RA, Munro MHG, Prinsep MR. Marine natural products. Nat Prod Rep, 2016; 33(3):382-431.

Blunt JW, Copp BR, Keyzers RA, Munro MHG, Prinsep MR. Marine natural products. Nat Prod Rep, 2017; 34(3):235-94.

Burrows JN, Chibale K, Wells TNC. The state of the art in antimalarial drug discovery and development. Curr Top Med Chem, 2011; 11(10):1226-54.

Campos P-E, Pichon E, Moriou C, Clerc P, Trépos R, Frederich M, Voogd ND, Hellio C, Gauvin-Bialecki A, Al-Mourabit A. New antimalarial and antimicrobial tryptamine derivatives from the marine sponge Fascaplysinopsis reticulata. Marine Drugs, 2019; 17(3):167.

Campos P-E, Wolfender J-L, Queiroz EF, Marcourt L, AlMourabit A, Frederich M, Bordignon A, Voogd ND, Illien B, GauvinBialecki A. Unguiculin A and Ptilomycalins E-H, antimalarial guanidine alkaloids from the marine sponge Monanchora unguiculata. J Nat Prod, 2017; 80(5):1404-10.

Carroll AR, Copp BR, Davis RA, Keyzers RA, Prinsep MR. Marine natural products. Nat Prod Rep, 2019; 36(1):122-73.

Chanthathamrongsiri N, Yuenyongsawad S, Wattanapiromsakul C, Plubrukarn A. Bifunctionalized amphilectane diterpenes from the sponge Stylissa cf. massa. J Nat Prod, 2012; 75(4):789-92.

Chin W, Moss D, Collins WE. The continuous cultivation of Plasmodium fragile by the method of Trager-Jensen. Am J Trop Med Hyg, 1979; 28(3):591-2.

Cui L, Mharakurwa S, Ndiaye D, Rathod PK, Rosenthal PJ. Antimalarial drug resistance: literature review and activities and findings of the ICEMR network. Am J Trop Med Hyg, 2015; 93(3 Suppl):57-68.

D'Ambrosio M, Guerriero A, Pietra F, Debitus C. Leucascandrolide A, a new type of macrolide: The first powerfully bioactive metabolite of calcareous sponges (Leucascandra caveolata, a new genus from the coral sea). Helvetica Chimica Acta, 1996; 79(1):51-60.

Davis RA, Buchanan MS, Duffy S, Avery VM, Charman SA, Charman WN, White KL, Shackleford DM, Edstein MD, Andrews KT, Camp D, Quinn RJ. Antimalarial activity of pyrroloiminoquinones from the Australian marine sponge Zyzzya sp. J Med Chem, 2012; 55(12):5851-8.

Desoubzdanne D, Marcourt L, Raux R, Chevalley S, Dorin D, Doerig C, Valentin A, Ausseil F, Debitus C. Alisiaquinones and alisiaquinol, dual inhibitors of Plasmodium falciparum enzyme targets from a new caledonian deep water sponge. J Nat Prod, 2008; 71(7):1189-92. 
Dondorp AM, Kager PA, Vreeken J, White NJ. Abnormal blood flow and red blood cell deformability in severe malaria. Parasitol Today (Regul Ed), 2000; 16(6):228-32.

Ebada SS, Edrada RA, Lin W, Proksch P. Methods for isolation, purification and structural elucidation of bioactive secondary metabolites from marine invertebrates. Nat Protoc, 2008; 3(12):1820-31.

Emsermann J, Kauhl U, Opatz T. Marine isonitriles and their related compounds. marine drugs, 2016; 14(1):16.

Farokhi F, Grellier P, Clément M, Roussakis C, Loiseau P, GeninSeward E, Kornprobst JM, Barnathan G, Wielgosz-Collin G. Antimalarial activity of axidjiferosides, new $\beta$-galactosylceramides from the African sponge axinyssa djiferi. Marine Drugs, 2013; 11(12):1304-15.

Fattorusso C, Persico M, Calcinai B, Cerrano C, Parapini S, Taramelli D, Novellino E, Romano A, Scala F, Fattorusso E, TaglialatelaScafati O. Manadoperoxides A-D from the Indonesian sponge Plakortis cfr. simplex. Further insights on the structure-activity relationships of simple 1,2-dioxane antimalarials. J Nat Prod, 2010; 73(6):1138-45.

Fattorusso E, Taglialatela-Scafati O. Marine antimalarials. Marine Drugs, 2009; 7(2):130-52.

Fattorusso E. Activity against Plasmodium falciparum of cycloperoxide compounds obtained from the sponge Plakortis simplex. J Antimicrob Chemother, 2002; 50(6):883-8.

Galeano E, Thomas OP, Robledo S, Munoz D, Martinez A. Antiparasitic bromotyrosine derivatives from the marine sponge verongula rigida. Marine Drugs, 2011; 9(10):1902-13.

Gochfeld DJ, Hamann MT. Isolation and biological evaluation of filiformin, plakortide $\mathrm{F}$, and plakortone $\mathrm{G}$ from the caribbean sponge Plakortis sp. J Nat Prod, 2001; 64(11):1477-9.

Gros E, Al-Mourabit A, Martin M-T, Sorres J, Vacelet J, Frederich M, Aknin M, Kashman Y, Gauvin-Bialecki A. Netamines H-N, Tricyclic alkaloids from the marine sponge Biemna laboutei and their antimalarial activity. J Nat Prod, 2014; 77(4):818-23.

Gros E, Martin M-T, Sorres J, Moriou C, Vacelet J, Frederich M, Aknin M, Kashman Y, Gauvin-Bialecki A, Al-Mourabit A. Netamines $\mathrm{O}-\mathrm{S}$, Five new tricyclic guanidine alkaloids from the madagascar sponge Biemna laboutei, and their antimalarial activities. Chem Biodivers, 2015; 12(11):1725-33.

Hu J-F, Schetz JA, Kelly M, Peng J-N, Ang KKH, Flotow H, Leong CY, Ng SB, Buss AD, Wilkins SP, Hamann MT. New antiinfective and human 5-HT2 receptor binding natural and semisynthetic compounds from the Jamaican sponge Smenospongia aurea. J Nat Prod, 2002; 65(4):476-80.

Ilias M, Ibrahim MA, Khan SI, Jacob MR, Tekwani BL, Walker LA, Samoylenko V. Pentacyclic ingamine alkaloids, a new antiplasmodial pharmacophore from the marine sponge petrosid Ng5 Sp5. Planta Med, 2012; 78(15):1690-7.

Jiménez-Romero C, Ortiz I, Vicente J, Vera B, Rodríguez AD, Nam S, Jove R. Bioactive Cycloperoxides isolated from the Puerto Rican sponge Plakortis halichondrioides. J Nat Prod, 2010; 73(10):1694-700.

Ju E, Latif A, Kong C-S, Seo Y, Lee Y-J, Dalal SR, Cassera MB, Kingston DGI. Antimalarial activity of the isolates from the marine sponge Hyrtios erectus against the chloroquine-resistant Dd2 strain of Plasmodium falciparum. Zeitschrift für Naturforschung C, 2018; 73(9-10):397-400.

Kelly M, Sim-Smith C, Stone R, Samaai T, Reiswig H, Austin W. New taxa and arrangements within the family latrunculiidae (Demospongiae, Poecilosclerida). Zootaxa, 2016; 4121(1):1.

Kirsch G, König GM, Wright AD, Kaminsky R. A new bioactive sesterterpene and antiplasmodial alkaloids from the marine sponge Hyrtios cf. erecta. J Nat Prod, 2000; 63(6):825-9.

Kurimoto S, Ohno T, Hokari R, Ishiyama A, Iwatsuki M, Ōmura S, Kobayashi J, Kubota T. Ceratinadins E and F, new bromotyrosine alkaloids from an Okinawan marine sponge pseudoceratina sp. Marine Drugs, 2018; 16(12):463.

Labaied M, Dagan A, Dellinger M, Gèze M, Egée S, Thomas SL, Wang C, Gatt S, Grellier P. Anti-plasmodium activity of ceramide analogs. Malar J, 2004; 3:49.
Laurent D, Jullian V, Parenty A, Knibiehler M, Dorin D, Schmitt S, Lozach O, Lebouvier N, Frostin M, Alby F, Maurel S, Doerig C, Meijer L, Sauvain M. Antimalarial potential of xestoquinone, a protein kinase inhibitor isolated from a Vanuatu marine sponge Xestospongia sp. Bioorg Med Chem, 2006; 14(13):4477-82.

Laville R, Thomas OP, Berrué F, Marquez D, Vacelet J, Amade P. Bioactive guanidine alkaloids from two Caribbean marine sponges. J Nat Prod, 2009; 72(9):1589-94.

Lazaro JEH, Nitcheu J, Mahmoudi N, Ibana JA, Mangalindan GC, Black GP, Howard-Jones AG, Moore CG, Thomas DA, Mazier D, Ireland CM, Concepcion GP, Murphy PJ, Diquet B. Antimalarial activity of Crambescidin 800 and synthetic analogues against liver and blood stage of plasmodium sp. J Antibiot, 2006; 59(9):583-90.

Lebouvier N, Jullian V, Desvignes I, Maurel S, Parenty A, DorinSemblat D, Doerig C, Sauvain M, Laurent D. Antiplasmodial activities of homogentisic acid derivative protein kinase inhibitors isolated from a Vanuatu marine sponge Pseudoceratina sp. Marine Drugs, 2009; 7(4):640-53.

Longeon A, Copp BR, Roué M, Dubois J, Valentin A, Petek S, Debitus C, Bourguet-Kondracki M-L. New bioactive halenaquinone derivatives from South Pacific marine sponges of the genus Xestospongia. Bioorg Med Chem, 2010; 18(16):6006-11.

Mani L, Jullian V, Mourkazel B, Valentin A, Dubois J, Cresteil T, Folcher E, Hooper JN, Erpenbeck D, Aalbersberg W, Debitus C. New antiplasmodial bromotyrosine derivatives from Suberea ianthelliformis lendenfeld, 1888. Chem Biodivers, 2012; 9(8):1436-51.

Marques J, Vilanova E, Mourão PAS, Fernàndez-Busquets X. Marine organism sulfated polysaccharides exhibiting significant antimalaria activity and inhibition of red blood cell invasion by Plasmodium. Sci Rep, 2016; 6(1):24368.

Mohammed R, Peng J, Kelly M, Hamann Mark T. Cyclic heptapeptides from the Jamaican sponge Stylissa caribica. J Nat Prod, 2006; 69(12):1739-44

Mudianta IW, Skinner-Adams T, Andrews KT, Davis RA, Hadi TA, Hayes PY, Garson MJ. Psammaplysin derivatives from the Balinese marine sponge Aplysinella strongylata. J Nat Prod, 2012; 75(12):2132-43.

Murtihapsari M, Salam S, Kurnia D, Darwati D, Kadarusman K, Abdullah FF, Herlina T, Husna MH, Awang K, Shiono Y, Azmi MN, Supratman U. A new antiplasmodial sterol from Indonesian marine sponge, Xestospongia sp. Nat Prod Res, 2019; 1-8.

Na M, Ding Y, Wang B, Tekwani BL, Schinazi RF, Franzblau S, Kelly M, Stone R, Li X-C, Ferreira D, Hamann MT. Anti-infective discorhabdins from a deep-water Alaskan sponge of the genus Latrunculia. J Nat Prod, 2010; 73(3):383-7.

Newman DJ, Cragg GM. Natural products as sources of new drugs over the last 25 years. J Nat Prod, 2007; 70(3):461-77.

Noedl H, Se Y, Schaecher K, Smith BL, Socheat D, Fukuda MM Evidence of artemisinin-resistant malaria in western Cambodia. N Engl J Med, 2008; 359(24):2619-20.

Orabi KY, El Sayed KA, Hamann MT, Dunbar DC, AlSaid MS, Higa T, Kelly M. Araguspongines K and L, new bioactive bis-1-oxaquinolizidine $\mathrm{N}$-Oxide Alkaloids from red sea specimens of Xestospongia exigua. J Nat Prod, 2002; 65(12):1782-5.

Orhan I, Şener B, Kaiser M, Brun R, Tasdemir D. Inhibitory activity of marine sponge-derived natural products against parasitic protozoa. Marine Drugs, 2010; 8(1):47-58.

Paul VJ, Puglisi MP, Ritson-Williams R. Marine chemical ecology. Nat Prod Rep, 2006; 23(2):153-80.

Paul VJ, Puglisi MP. Chemical mediation of interactions among marine organisms. Nat Prod Rep, 2004; 21(1):189-209.

Perdicaris S, Vlachogianni T, Valavanidis A. Bioactive natural substances from marine sponges: new developments and prospects for future pharmaceuticals. Nat Prod Chem Res, 2013; 1(3):114.

Prakasa Rao TS, Sarma NS, Murthy YLN, Kantamreddi VSSN, Wright CW, Parameswaran PS. New polyhydroxy sterols from the marine sponge Callyspongia fibrosa (Ridley \& Dendly). Tetrahedron Letters, 2010; 51(27):3583-6. 
Qaralleh H. Chemical and bioactive diversities of marine sponge Neopetrosia. Bangladesh J Pharmacol, 2016; 11(2):433-52.

Radwan MM, Wanas AS, Fronczek FR, Jacob MR, Ross SA. Polybrominated diphenyl ethers from the marine organisms Lendenfeldia dendyi and Sinularia dura with anti-MRSa activity. Med Chem Res, 2015; 24(9):3398-404.

Rama Rao M, Faulkner DJ. Isotactic polymethoxydienes from the Philippines sponge Myriastra clavosa. J Nat Prod, 2002; 65(8):1201-3.

Rao KV, Donia MS, Peng J, Garcia-Palomero E, Alonso D, Martinez A, Medina M, Franzblau SG, Tekwani BL, Khan SI, Wahyuono S, Willett KL, Hamann MT. Manzamine B and E and Ircinal A related alkaloids from an Indonesian Acanthostrongylophora sponge and their activity against infectious, tropical parasitic, and Alzheimer's diseases. J Nat Prod, 2006; 69(7):1034-40.

Rao KV, Kasanah N, Wahyuono S, Tekwani BL, Schinazi RF, Hamann MT. Three new manzamine alkaloids from a common Indonesian sponge and their activity against infectious and tropical parasitic diseases. J Nat Prod, 2004; 67(8):1314-8.

Samoylenko V, Khan SI, Jacob MR, Tekwani BL, Walker LA, Hufford CD, Muhammad I. Bioactive (+)-Manzamine $A$ and (+)-8-Hydroxymanzamine a tertiary bases and salts from Acanthostrongylophora Ingens and their preparations. Nat Prod Commun, 2009; 4(2):185-92.

Scala F, Fattorusso E, Menna M, Taglialatela-Scafati O, Tierney M, Kaiser M, Tasdemir D. Bromopyrrole alkaloids as lead compounds against protozoan parasites. Marine Drugs, 2010; 8(7):2162-74.

Setyowati EP, Jenie UA, Sudarsono, Kardono LBS, Rahmat R. Theonellapeptolide Id: structure identification of cytotoxic constituent from Kaliapsis sp. Sponge (Bowerbank) collected from West Bali Sea Indonesia. J Biol Sci, 2009; 9(1):29-36.

Setyowati EP, Pratiwi S, Hertiani T, Samara O. Bioactivity of Fungi Trichoderma reesei associated with sponges Stylissa flabelliformis collected from National Park West Bali, Indonesia. J Biol Sci, 2017a; 17(8):362-8.

Setyowati EP, Pratiwi SUT, Purwantiningsih P, Samara O. Antimicrobial activity and identification of fungus associated Stylissa flabelliformis sponge collected from Menjangan Island West Bali National Park, Indonesia. Indonesian J Pharm, 2017b; 29(2):66.

Simoes-Pires C, Hostettmann K, Haouala A, Cuendet M, Falquet J, Graz B, Christen P. Reverse pharmacology for developing an anti-malarial phytomedicine. The example of Argemone mexicana. Int J Parasitol Drugs Drug Resist, 2014; 4(3):338-46.

Sipkema D, Franssen MCR, Osinga R, Tramper J, Wijffels RH. Marine sponges as pharmacy. Mar Biotechnol, 2005; 7(3):142.

Sirirak T, Kittiwisut S, Janma C, Yuenyongsawad S, Suwanborirux $\mathrm{K}$, Plubrukarn A. Kabiramides J and K, Trisoxazole macrolides from the sponge Pachastrissa nux. J Nat Prod, 2011; 74(5):1288-92.
Soest RWM van (Amsterdam U (Netherlands) I for S and PB, Desqueyroux-Faundez R, Wright AD, Koenig GM. Cymbastela hooperi sp. nov. (Halichondrida: Axinellidae) from the Great Barrier Reef, Australia Bulletin van het Koninlijk Belgisch Instituut voor Natuurwetenschappen Biologie, 1996. [ONLINE]. Available via http://agris.fao.org/agris-search/ search.do?recordID=BE1997001470 (Accessed 31 January 2020).

Tasdemir D, Topaloglu B, Perozzo R, Brun R, O’Neill R, Carballeira NM, Zhang X, Tonge PJ, Linden A, Rüedi P. Marine natural products from the Turkish sponge Agelas oroides that inhibit the enoyl reductases from Plasmodium falciparum, Mycobacterium tuberculosis and Escherichia coli. Bioorg Med Chem, 2007; 15(21):6834-45.

Thomas TRA, Kavlekar DP, LokaBharathi PA. Marine drugs from sponge-microbe association-a review. Marine Drugs, 2010; 8(4):1417-68.

Ueoka R, Nakao Y, Kawatsu S, Yaegashi J, Matsumoto Y, Matsunaga S, Furihata K, Soest RWM van, Fusetani N. Gracilioethers A-C, Antimalarial metabolites from the marine sponge Agelas gracilis. J Org Chem, 2009; 74(11):4203-7.

World Health Organization. World malaria report, 2018 [ONLINE]. Available via http://www.who.int/malaria/publications/worldmalaria-report-2018/en/ (Accessed 3 July 2019).

Wright AD, Lang-Unnasch N. Diterpene Formamides from the tropical marine sponge Cymbastela hooperi and their antimalarial activity in vitro. J Nat Prod, 2009; 72(3):492-5.

Xu M, Andrews KT, Birrell GW, Tran TL, Camp D, Davis RA, Quinn RJ. Psammaplysin H, a new antimalarial bromotyrosine alkaloid from a marine sponge of the genus Pseudoceratina. Bioorg Med Chem Lett, 2011; 21(2):846-8.

Yang X, Davis RA, Buchanan MS, Duffy S, Avery VM, Camp D, Quinn RJ. Antimalarial bromotyrosine derivatives from the Australian marine sponge Hyattella sp. J Nat Prod, 2010; 73(5):985-7.

Zhang K, Bangs JD, Beverley SM. Sphingolipids in parasitic protozoa. Adv Exp Med Biol, 2010; 688:238-48.

How to cite this article:

Hikmawan BD, Wahyuono S, Setyowati EP. Marine sponge compounds with antiplasmodial properties: Focus on in vitro study against Plasmodium falciparum. J Appl Pharm Sci, 2020; 10(05):142-157. 